\title{
Understanding the Role of Additives in Improving the Performance of Bulk Heterojunction Organic Solar Cells
}

\author{
Wei Chen ${ }^{1,2}$, Seth B. Darling ${ }^{2,3}$ \\ 1. Materials Science Division, Argonne National Laboratory, Argonne, Illinois, 60439, USA \\ 2. Institute for Molecular Engineering, the University of Chicago, Chicago, Illinois, 60637, USA \\ 3. Center for Nanoscale Materials, Nanoscience and Technology Division, Argonne National Laboratory, \\ Argonne, Illinois, 60439, USA
}

Organic solar cells based on the bulk heterojunction (BHJ) represent one of the most promising technologies for next-generation solar energy conversion due to their low-cost and scalability. In the last ten years, the highest power conversion efficiency (PCE) obtained from organic solar cells has risen from 2.5 to $12 \%{ }^{1}$ Controlling BHJ morphology is essential for achieving high PCE. Processing BHJ layers with solvent additives has proven to be an effective strategy toward achieving BHJ morphology correlated with high efficiency. As examples, the incorporation of a small percentage of solvent additives, such as $3 \mathrm{v} \%$ and $0.25 \mathrm{v} \%$ of 1,8-diiodooctane (DIO) in PTB7:PC $71 \mathrm{BM}^{2}$ and $\mathrm{DTS}\left(\mathrm{PTTh}_{2}\right)_{2}: \mathrm{PC}_{71} \mathrm{BM}^{3}$, respectively, results in a nearly doubling of device efficiency. However, the mechanism underlying their role in improving power conversion efficiency (PCE) has remained elusive. The general picture of a correlation between solvent constituents and the morphology within the deposited active layer film is clear, but the nature of this correlation is unknown - and it must be deciphered in order to adopt a more rational approach to improving device function. Using a combination of characterization techniques including x-ray and neutron scattering as well as advanced electron microscopy, we probe the influence of additives on active layer morphology in several state-ofthe-art BHJ blend systems. Through the integrated analysis of real- and reciprocal-space data, we uncover new insights into the role of additives that can be generalized across multiple materials.

In this work, in order to explore general principles of the impact of additives on organic solar cells, several high-performance materials were selected for study. PTB7 ${ }^{2}$ and PBDTP-DPP ${ }^{4}$ represent topperformers among conjugated polymers in polymer:fullerene $\mathrm{BHJ}$ devices and $\mathrm{DTS}\left(\mathrm{PTTh}_{2}\right)_{2}{ }^{3}$ is among the leading solution-processed small molecule materials when blended with fullerenes. Morphology in these high-performance systems varies quantitatively, but each system shares some common structural motifs. Key among these is a hierarchical morphology comprised of crystallites several nanometers in scale, aggregates of crystallites spanning tens of nanometers, and donor-rich/acceptor-rich regions nearly an order of magnitude larger (Figure 1). The superior performance of polymer:fullerene BHJ solar cells is attributed to hierarchical nanomorphologies with optimum crystallinity and nanoscale intermixing of copolymers with fullerenes. ${ }^{5}$ In addition, these morphologies are not equilibrium structures, meaning that the kinetic pathway by which they are created is central. The solvent additives induce the change in the formation mechanism of polymer:fullerene nanomorphologies in the process of film casting. Additives have direct impact on the interactions between the molecular constituents in a BHJ blend, and those interactions can be explored using techniques such as small angle neutron scattering (SANS). Using this approach, we shed light on how additives position the system in a different region of the phase diagram in solution, where important aspects of the morphology take form. Together, one can readily see a significant qualitative shift in morphology starting in the solution and solidifying in the film when additives are present. This shift is observed in each of the systems studied. This new insight into the role of additives provides a pathway for rational design of optimized 
morphology in OPV active layers.

References: (Times New Roman, 12 pt, left justified)

[1] M. A.Green, K. Emery, Y. Hishikawa, W. Warta, E. D. Dunlop, Prog. Photovolt: Res. Appl. 2015, 23,1 .

[2] Y. Liang, Z. Xu, J. Xia, S.-T.Tsai, Y. Wu, Li, G.; Ray, C.; Yu, L. Adv. Mater. 2010, 22, E135.

[3] Y. M. Sun, G. C. Welch, W. L. Leong, C. J. Takacs, G. C. Bazan, A. J. Heeger, Nature Mater. 2012, $11,44$.

[4] L. Dou, J. You, J. Yang, C.-C. Chen, Y. He, S. Murase, T. Moriarty, K. Emery, G. Li, Y. Yang, Nature Photon. 2012, 6, 180.

[5] W. Chen, T. Xu, F. He, W. Wang, C. Wang, J. Strzalka, Y. Liu, J. Wen, D. J. Miller, J. Chen, K. Hong, L. Yu, S. B. Darling, Nano Lett. 2011, 11, 3707.

[6] This work was supported by the U.S. Department of Energy, Office of Science, Basic Energy Sciences, Materials Sciences and Engineering Division. Use of the Advanced Photon Source (APS), the Electron Microscopy Center (EMC) for Materials Research, and the Center for Nanoscale Materials (CNM) at Argonne National Laboratory was supported by the U.S. Department of Energy, Office of Science, Office of Basic Energy Sciences, under Contract No. DE-AC02-06CH11357. The ALS at Lawrence Berkeley National Laboratory is supported by the Director, Office of Science, Office of Basic Energy Sciences, of the U.S. Department of Energy under Contract No. DE-AC02-05CH11231. A portion of this research was conducted at the Center for Nanophase Materials Sciences (CNMS), the High Flux Isotope Reactor (HFIR), and Spallation Neutron Source (SNS), which are sponsored at Oak Ridge National Laboratory by the Office of Basic Energy Sciences, U.S. Department of Energy. We also acknowledge the support of the National Institute of Standards and Technology (NIST), U.S. Department of Commerce, in providing the neutron research facilities used in this work. This work utilized NCNR facilities supported in part by the National Science Foundation under Agreement No. DMR-0454672.

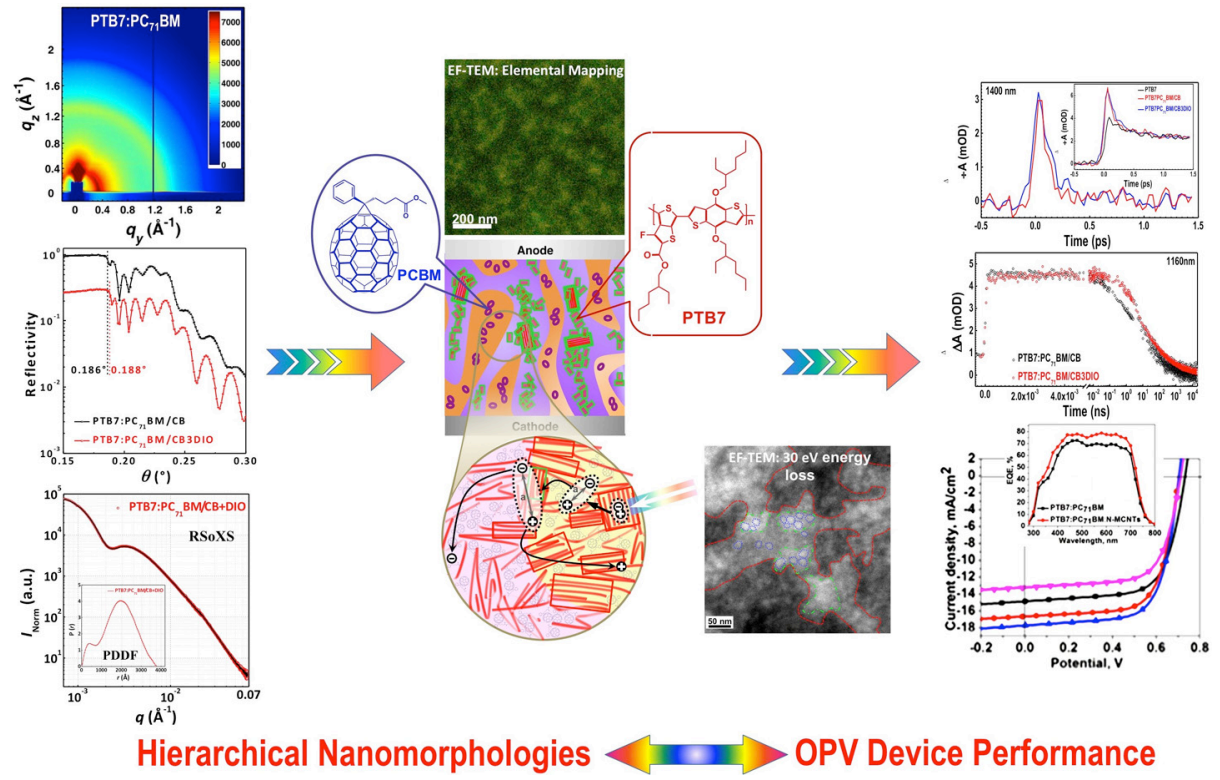

Figure 1. Correlating hierarchical nanomorphologies to OPV performance especially promotion of exciton dissociation, in polymer:fullerene thin film solar cells. 